Short reports

\title{
Face mask physiotherapy in cystic fibrosis
}

\author{
J C TYRRELL, E J HILLER, AND J MARTIN
}

City Hospital, Nottingham

SUMMARY The use of the 'PEP' mask with forced expiratory coughing was compared with conventional physiotherapy over a one month period. No difference was shown in symptom scores, sputum production, or simple lung function tests. The mask was well accepted and allowed independent treatment by older patients.

Regular chest physiotherapy with postural drainage and percussion is one of the most important aspects of treatment in cystic fibrosis. Although many studies fail to show any improvement in simple lung function tests after a single treatment, withdrawal of physiotherapy causes deterioration, which can be reversed after resumption of treatment. ${ }^{1}$

Physiotherapy is usually performed by a parent twice daily, which is time consuming, and compliance is often poor. Older children and adults need to become independent. Short term studies with adult patients have shown that a combination of postural drainage and forced expiratory coughing is as effective as conventional physiotherapy in mobilising sputum. ${ }^{2}$ Danish workers have developed a face mask incorporating a fixed expiratory resistance, known as the 'PEP' (positive expiratory pressure) mask, which is used in conjunction with forced expiratory coughing but without postural drainage. A single treatment was shown to result in greater sputum production and higher transcutaneous oxygen measurements than a single postural drainage session with percussion or forced expiratory coughing in the sitting position. ${ }^{3}$ Prolonged treatment over six to nine months was associated with a significant fall in residual volume, other lung function tests remaining unchanged. ${ }^{4}$ These authors believe that the technique works by allowing more air to enter the small airways through collateral channels during inspiration than escapes during expiration, so that a pressure gradient builds up behind sputum plugs, forcing the secretions towards the larger airways where they can be cleared more easily. ${ }^{4}$ This technique is used widely in Denmark with good patient compliance.

We investigated the use of a commercially available 'PEP' mask (Astra Meditec) in a group of older children with cystic fibrosis.

\section{Method}

We followed the manufacturer's recommendations for 'PEP' treatment. Using a manometer, an appropriate resistor is attached to the outflow valve such that the patient is breathing against a pressure of 10-15 cm water (Fig. 1). The manometer can then be removed from the system. PEP treatment consists of 10 breaths through the mask followed by forced expiratory coughing in the sitting position. This is repeated for 20 minutes twice daily.

We compared one month of PEP treatment with one month of standard physiotherapy (two sessions of postural drainage, percussion, and coughing daily) in random order. Diary cards were used to record symptoms (sleep, cough, wheeze, activity, and sputum production, with a maximum score of

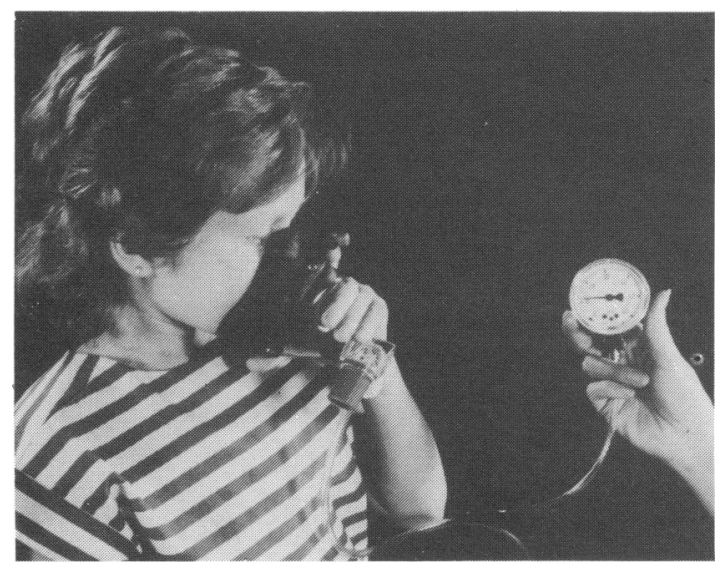

Fig. 1 Using the PEP mask. 
13). Those children who showed airway reversibility with salbutamol were asked to use it before treatment throughout the whole study.

The children were seen at the beginning and end of each study month. At these visits we recorded peak expiratory flow rate (PEFR), forced expiratory volume in 0.75 second $\left(\mathrm{FEV}_{0.75}\right)$, and forced vital capacity (FVC) using a Vitalograph, before and 20 and 90 minutes after a single treatment under supervision, either physiotherapy performed by a parent or treatment with the PEP mask. We weighed the amount of sputum expectorated at

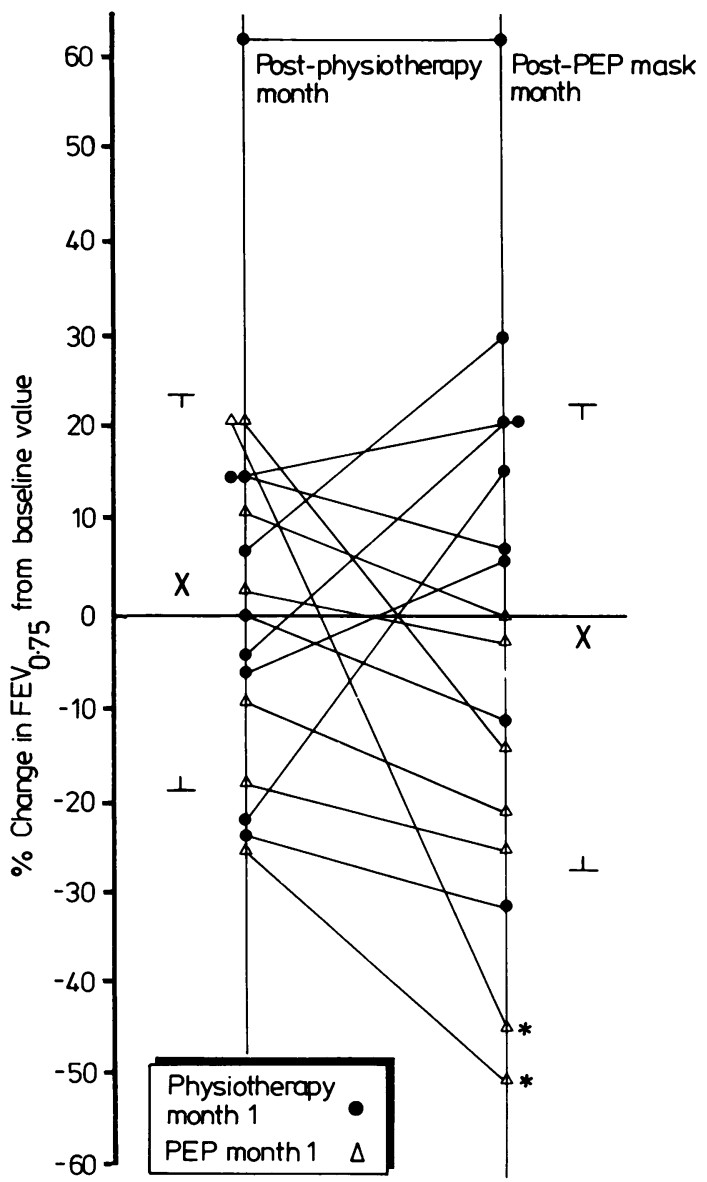

Fig. $2 \%$ Changes in FEV 0.75 after one month of treatment with 'PEP' mask and one of standard physiotherapy compared with initial baseline measurement. Mean (SD) values shown to left and right of changes.

*Two children who deteriorated during the month of treatment with PEP improved during the subsequent month of treatment with physiotherapy these sessions. The results were compared statistically using Student's $t$ test.

\section{Results}

Nineteen children initially agreed to take part in the study, but three were excluded because of noncompliance. There were seven boys and nine girls, with an age range of 10-18 years and a mean age of 13. They had mild to moderate disease with Shwachman scores ranging from 47-85 (mean 62, 'normal' score $=100$ ), and Chrispin-Norman $x$ ray scores ranging from 3-20 (mean 12.6, normal $x$ ray $=0$, most changes $=38) .56$

After a single supervised physiotherapy and PEP treatment there was a similar small increase in $\mathrm{FEV}_{0.75}$ and FVC (prephysiotherapy $\mathrm{FEV}_{0.75}=$ $53 \pm 21 \%$, mean predicted value for height, 20 minutes post-treatment $\mathrm{FEV}_{0.75}=58 \pm 21 \%$, pre-PEP mask treatment $\mathrm{FEV}_{0.75}=50 \pm 21 \%, 20$ minutes posttreatment $\mathrm{FEV}_{0.75}=53 \pm 22 \%$, not significant). Mean sputum production was $8.2 \mathrm{~g}$ and $8.1 \mathrm{~g}$ for the physiotherapy and PEP mask sessions, respectively (range $=0-44.7 \mathrm{~g}$ ). No significant difference in $\mathrm{FEV}_{0.75}, \mathrm{FVC}$, or PEFR at the end of the two treatment periods was shown (Fig. 2). Mean (range) daily diary scores were $3.5(0-9.4)$ during the physiotherapy month and $3.7(0-9)$ in the PEP month.

Antibiotic consumption was the same for each month. Two children did deteriorate in the month with PEP treatment but improved during the subsequent month with physiotherapy. Comments about the mask were generally favourable. At the end of the study we offered the option of continuing treatment with the mask to those children who wished to. Six months later nine children were using it exclusively, four were using it in addition to conventional physiotherapy, and three had found no benefit from it. There was no significant change in a single measurement of $\mathrm{FEV}_{0.75}, \mathrm{FVC}$, or their chest $x$ ray scores.

\section{Discussion}

We have shown that the use of a PEP mask with forced expiratory coughing was as effective as conventional physiotherapy over a short period. The initial enthusiasm of parents and children who were impressed by the degree of independence and lessening of family tensions achieved was not maintained by all. Some parents felt guilty at not doing the physiotherapy themselves and tended to revert to conventional treatment when the child had a cold. We gained the impression that children producing moderate or large amounts of sputum 
were least happy with the PEP mask, complaining that their chests did not always feel clear after treatment. Independent treatment of this sort requires motivation and self discipline on the child's part, as well as parental supervision, and is probably not suitable for most children under 10 years. For some teenagers it is a valuable and acceptable form of treatment, allowing considerable independence. Older patients might manage satisfactorily with postural drainage and forced expiratory coughing alone-we intend to compare this with PEP mask treatment.

We thank Astra Meditec for the loan of the PEP masks. JT is supported by the Cystic Fibrosis Research Trust.

References

${ }^{1}$ Desmond KJ, Schwent WF, Thomas E, Beaudry PH,
Coates AL. Immediate and long term effects of chest physiotherapy in patients with cystic fibrosis. $J$ Pediatr 1983;103:538-42.

2 Pryor A, Webber BA, Hodson ME, Batten JC. Evaluation of the forced expiration technique as an adjunct to postural drainage in treatment of cystic fibrosis. $\mathrm{Br}$ Med J 1979;ii:417-8.

${ }^{3}$ Falk M, Kelstrup M, Andersen J, et al. Improving the Ketchup bottle method with positive expiratory pressure, PEP, in cystic fibrosis. Eur J Respir Dis 1984;65:423-32.

4 Tønnesen P, Støvring S. Positive expiratory pressure (PEP) as lung physiotherapy in cystic fibrosis-a pilot study. Eur J Respir Dis 1984;65:419-22.

5 Shwachman H, Kulczycki L. Long term study of 105 patients with cystic fibrosis. Am J Dis Child 1958;96:6-15.

${ }^{6}$ Chrispin A, Norman A. The systematic evaluation of the chest radiograph in cystic fibrosis. Pediatr Radiol 1974;2:101-6.

Correspondence to Dr J C Tyrrell, Ward A2, City Hospital, Hucknall Road, Nottingham NG5 1PB, England.

Received 26 February 1986

\title{
Severe neonatal pertussis treated by salbutamol
}

\author{
A Y-C TAM AND C-Y YEUNG
}

Department of Paediatrics, Queen Mary Hospital, University of Hong Kong, Hong Kong

SUMmary Two neonates with severe culture proven pertussis were successfully treated with salbutamol $0.5 \mathrm{mg}$ three times daily. There was a demonstrable decrease in the total number of coughing spasms, their mean duration and severity. In one baby this was shown by the transcutaneous oxygen tension measurements.

Vaccination against pertussis has done much to decrease the incidence and mortality of the disease. Complete eradication is still not possible, however, with the present vaccine. Moreover, the epidemiology of the disease has changed, in that it now affects more young infants who are too young to receive the immunisation. ${ }^{1}$ Although treatment with erythromycin successfully kills the bacterium, it can alleviate the coughing spasms only if given very early. In young infants coughing spasms are not only distressing but also related to complications. Hence the search for treatment of the cough spasms of pertussis is still continuing. Beta adrenergic blockade in pertussis has been shown in children. ${ }^{2}$ Various workers have shown the effectiveness of salbutamol, a beta 2 agonist, in alleviating the coughing spasms in pertussis. ${ }^{3}$ We report the success of using salbutamol in two neonates with culture proven Bordetella pertussis infection.

\section{Case reports}

Case 1. A 14 day old girl was admitted with a one $\overrightarrow{\overrightarrow{0}}$ day history of coughing spasms associated with $\exists$ cyanosis. In between the spasms she remained pink and well. Her white blood cell count was $30 \cdot 8 \times 10^{9} / 1$ (lymphocytes $70 \%$ ). Pernasal swab grew Bordetella pertussis. Treatment with erythromycin was started on admission. Salbutamol was also given at a dose of $0.5 \mathrm{mg}$ three times daily. The infant was nursed in the intensive care unit with an attending nurse by $\delta$ the cot side who would record in detail the onset and $₹$ duration of coughing spasms. Manipulation and pharyngeal suction was kept to a minimum. Oxygen was administered through an oxygen funnel during the paroxysms. The child was kept in the intensive $\vec{N}$ care unit for a total of 13 days. The number of 0 coughing spasms decreased slowly from 31 on day $1 \stackrel{N}{N}$ of admission to 20 on day 4 and further to 10 on day 8. The mean duration of coughing spasms, however, was markedly decreased by treatment with salbuta- 0

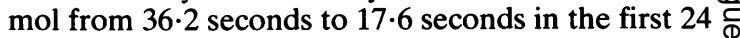
hours of administration of salbutamol (Figure). The $\stackrel{\oplus}{\rightarrow}$ treatment with salbutamol was continued for a total 0 of 10 days. Her subsequent recovery was uneventful.

Case 2. A 20 day old girl had a cough for two days that was deteriorating. On admission she was having 